\title{
Recurrent laryngeal nerve injury assessment by intraoperative laryngeal ultrasonography: a prospective diagnostic test accuracy study
}

\author{
Andrius Rybakovas ${ }^{1}$, Augustinas Bausys ${ }^{1}$, Andrius Matulevicius², Gytis Zaldokas², Mindaugas Kvietkauskas ${ }^{1}$, \\ Gintautas Tamulevicius ${ }^{3}$, Virgilijus Beisa ${ }^{1}$, Kestutis Strupas ${ }^{1}$ \\ ${ }^{1}$ Clinic of Gastroenterology, Nephrourology and Surgery, Institute of Clinical Medicine, Faculty of Medicine, Vilnius University, Vilnius, \\ Lithuania \\ ${ }^{2}$ Center of Ear, Nose and Throat, Vilnius University Hospital Santaros Clinics, Vilnius, Lithuania \\ ${ }^{3}$ Institute of Data Science and Digital Technologies, Vilnius University, Vilnius, Lithuania
}

Videosurgery Miniinv 2019; 14 (1): 38-45 DOI: https://doi.org/10.5114/wiitm.2018.80066

\begin{abstract}
Introduction: Recurrent laryngeal nerve injury is one of the major complications related to thyroid surgery. Intraoperative recurrent laryngeal nerve functional status monitoring is becoming a standard part of thyroid surgery. However, the current methods for intraoperative nerve functional status assessment are associated with a demand for specialized devices and increased costs.

Aim: To assess the validity of a new method - intraoperative laryngeal ultrasonography - for prediction of recurrent laryngeal nerve injury.

Material and methods: This prospective diagnostic test accuracy study included 112 patients undergoing thyroid surgery in Vilnius University Hospital Santaros Clinics. Neurostimulation combined with laryngeal ultrasonography and laryngeal palpation was performed intraoperatively to evaluate recurrent laryngeal nerve functional status. Recurrent laryngeal nerve injury was confirmed by laryngoscopy, which was performed on the first postoperative day and considered to be the gold standard method.

Results: Data on 112 consecutive patients and 200 nerves at risk were collected. The temporary vocal cord palsy rate was 5.4\% per patient and 3\% per nerve at risk. No permanent palsy or bilateral injury cases were registered in the study cohort. Laryngeal ultrasound sensitivity counted per nerve at risk was $83.3 \%$, specificity $97.2 \%$, accuracy $96.4 \%$, positive predictive value $62.5 \%$ and negative predictive value $99 \%$.

Conclusions: Laryngeal ultrasonography is a feasible new technique for accurate intraoperative recurrent laryngeal nerve injury evaluation.
\end{abstract}

Key words: thyroid surgery, recurrent laryngeal nerve, vocal cord palsy, laryngeal ultrasound.

\section{Introduction}

The number of thyroid surgery cases is steadily increasing [1]. While the safety of thyroidectomy has improved, recurrent laryngeal nerve (RLN) palsy is a widely acknowledged complication in thyroid surgery. Reported incidence rates vary from $0 \%$ to $38.4 \%$ and generally are accepted as underreported $[2,3]$. Though voice and speech have very important roles in human social life and professional performance, bilateral laryngeal nerve injury is a partic- 
ularly pressing concern for a surgeon as it can inflict such serious ill effects as breathing difficulties, reintubation, tracheostomy or even death. Every surgeon should be aware of how to avoid bilateral RLN palsy. Most surgeons agree that RLN should be identified in all cases. The advantage of this approach has been proven by many studies [4-6]. Furthermore, identifying the functional status of an RLN during thyroid surgery is of paramount significance, as in most cases the injured nerve seems visually intact $[7,8]$. Injury identification intraoperatively is crucial, because it can change the operative strategy: the operation on the second side can be canceled, two-stage thyroidectomy planned and bilateral nerve injury avoided [9]. Intraoperative neuromonitoring (IONM) has gained general acceptance among thyroid surgeons. The most common method in use is a special endotracheal tube with embedded electrodes for electromyographic recordings. Despite the widespread popularity of this technique, disadvantages such as a substantial learning curve, increased cost, additional setup time, equipment dysfunctions related to tube malpositioning, low sensitivity, and positive predictive value are repeatedly mentioned [10-13]. Moreover, the possibility to reliably predict RLN functional status intraoperatively is sometimes seriously doubted [14, 15]. An alternative simple and inexpensive technique of vocal cord (VC) mobility evaluation with similar predictive values to IONM is finger palpation of the cricoarytenoid muscle or so-called laryngeal palpation (LP) [16-22]. A new promising technique, gaining in popularity in the perioperative setting, is laryngeal ultrasonography (LU) [23-27]. To our knowledge, there has been no attempt to assess the feasibility of $L U$ as an intraoperative technique to date. Keeping in mind such important points as the less invasive and more objective character of LU technique, it could be considered as a very promising method for intraoperative RLN functional status assessment which could be used in both conventional and minimally invasive thyroid surgery.

\footnotetext{
Aim

We designed this study to evaluate the efficacy of intraoperative LU for RLN functional status verification. Another objective of this study was to compare the predictive values of the intraoperative $L U$ and LP methods.
}

\section{Material and methods}

\section{Patients' selection}

All patients who underwent thyroid surgery between March 2016 and December 2017 in Vilnius University Hospital Santaros Clinics were screened for inclusion in this prospective diagnostic test accuracy study. The protocol of the study was approved by the Vilnius regional bioethics committee. All patients aged 18 years or older who had thyroid pathology and were scheduled for thyroid surgery were eligible. Exclusion criteria were as follows: pregnancy, refusal to participate in the study, and presence of RLN palsy before the surgery.

\section{Study protocol}

All patients prior to the inclusion in the study underwent preoperative laryngeal examination by indirect or fiber optic laryngoscopy for VC mobility evaluation. Those patients who had no evidence of RLN palsy were enrolled in the study and underwent thyroid surgery. In all cases, surgery and all intraoperative tests for RLN functional status evaluation were performed by a single surgeon (A.R.). To reduce errors in LU interpretation, the surgeon was additionally trained in the Instructional Course of Transcutaneous Laryngeal Ultrasonography at the first World Congress of Neural Monitoring in Thyroid and Parathyroid Surgery (September 2015, Krakow) held by the International Neural Monitoring Study Group. Intraoperative LP and LU were performed as described below. Laryngoscopy was performed in all patients on the first postoperative day. Follow-up laryngoscopy was reserved for those patients who were diagnosed with VC palsy on the first postoperative laryngeal examination. Follow-up checkups were performed every 3 months during the first year after the surgery. If VC palsy was present for more than 1 year it was considered as permanent RLN palsy.

\section{Recurrent laryngeal nerve and vagus nerve stimulation}

Both the vagus nerve and RLN were stimulated during LP and LU at the end of the surgery of each side. The nerve stimulator Stimuplex HNS 12 (BBraun, Melsungen, Germany) was used for stimulation with stimulus amplitude $1 \mathrm{~mA}$ and stimulus frequency $2 \mathrm{~Hz}$. No neuromuscular blocking agents were used after the induction of anesthesia. The proper functioning of the neural stimulator was tested in each 
Andrius Rybakovas, Augustinas Bausys, Andrius Matulevicius, Gytis Zaldokas, Mindaugas Kvietkauskas, Gintautas Tamulevicius, Virgilijus Beisa, Kestutis Strupas

case on the muscular tissue of the surgical field before direct nerve stimulation. As the international neural monitoring study group has emphasized the importance of vagal nerve stimulation for accurate nerve prognostication, the vagus nerve was dissected and visually identified in all cases [28].

\section{Laryngeal palpation technique}

For laryngeal palpation, intraoperative neurostimulation was performed as described above and the functional status of the RLN was assessed by palpating the post-cricoid region of the larynx, and sensing posteior cricoarytenoid muscle contraction, the so-called "laryngeal twitch resresponse" [16].

\section{Laryngeal ultrasound technique}

For the utrasound system, BK Flex Focus 8008815 , 4-10 MHz was used for ultrasound evaluation. The transcutaneous laryngeal ultrasonography tech- nique was adopted for intraoperative use, applying acoustic gel and covering the probe with a sterile sleeve. To improve surface contact warm sterile saline was used on the outer surface of the probe cover. Anterior-approach and lateral-approach LU were used to increase the VC "assessability" rate; the techniques were performed as described elsewhere $[25,26]$ (Photo 1).

Using the anterior-approach a transducer was placed on the skin above the surgical wound at the middle of the thyroid cartilage. In the case of the lateral approach, the transducer was placed on the lateral surface of the thyroid cartilage lamina. The 3 most commonly described sonographic landmarks were chosen for ultrasonographic visualization: false cords, true cords, and arytenoids. Intraoperative LU examination was defined as assessable if 1 or more landmarks were identified. The anterior-approach LU was performed first, whereas the lateral-approach LU was performed only when visualization using the
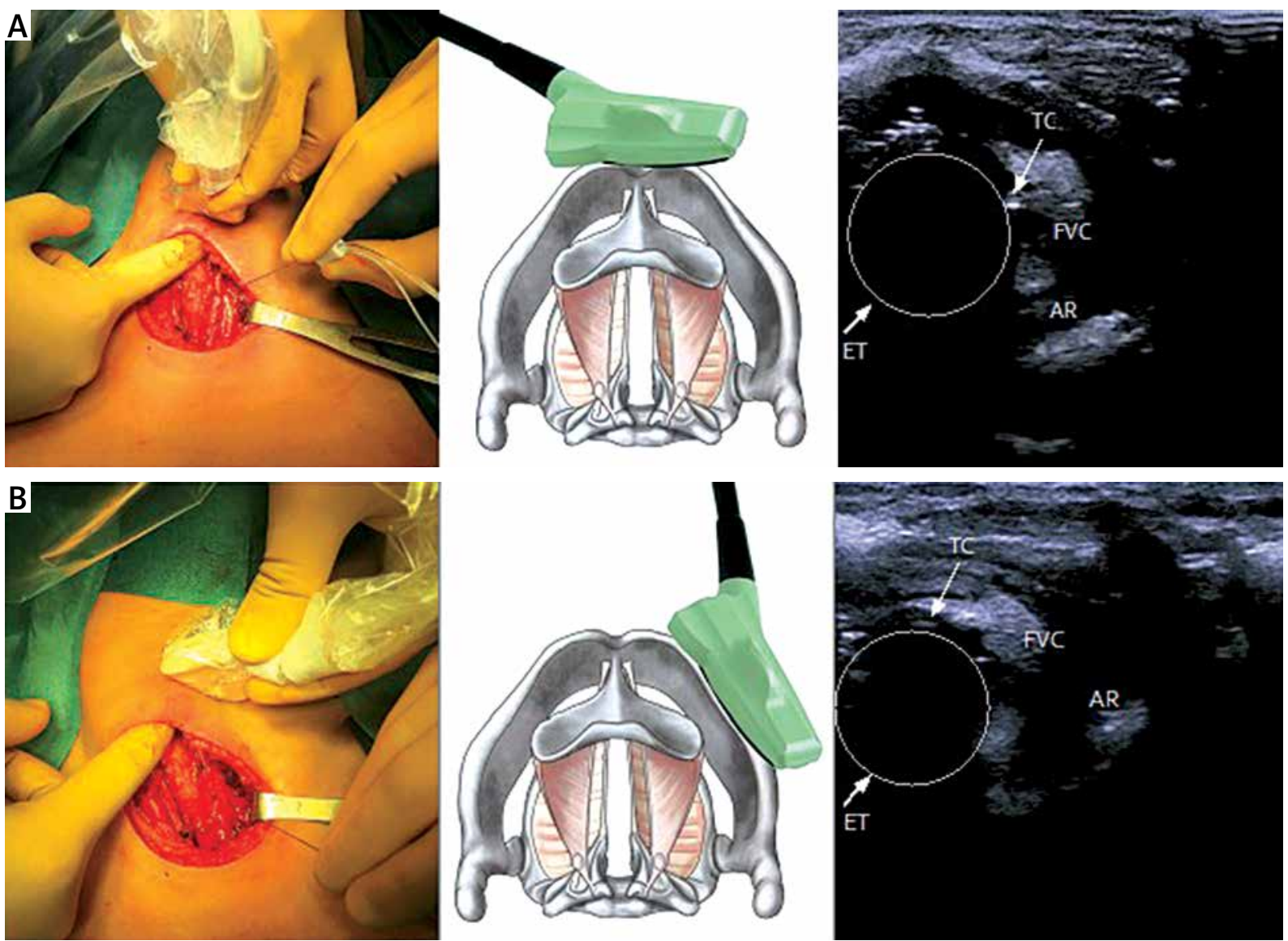

Photo 1. Anterior-approach laryngeal ultrasound and lateral-approach laryngeal ultrasound

$A R$ - arytenoids, FVC - false vocal cord, TC - true vocal cord, ET - endotracheal tube. 


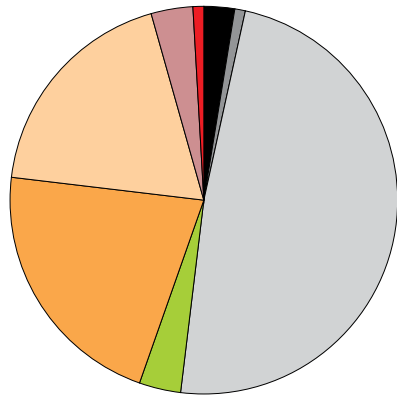

Total - 112
- Thyroidectomy + parathyroidectomy (2.7\%)

$\square$ Thyroidectomy + parathyroidectomy + two sided lymphadenectomy $(0.9 \%)$

$\square$ Thyroidectomy (48.2\%)

$\square$ Thyroidectomy + modified radical lymphadenectomy (3.6\%)

$\square$ Lobisthmectomy (21.4\%)

$\square$ Thyroidectomy + one sided central lymphadenectomy (18.7\%)

$\square$ Thyroidectomy + two sided central lymphadenectomy (3.6\%)

$\square$ Removal of residual tissue $(0.9 \%)$

Figure 1. Indications for surgery

anterior-approach technique proved unsuccessful. The observed movement in any of 3 landmarks was considered as sufficient proof of normal RLN function [23].

\section{Definitions and statistical analysis}

If both the LU and postoperative laryngoscopy confirmed RLN palsy, the case was defined as true positive. If both tests refuted RLN palsy the case was defined as true negative. If movement was observed in any of the 3 chosen sonographic landmarks on intraoperative LU, but postoperative laryngoscopy revealed VC palsy, the case was interpreted as false negative, and vice versa, if no movement was observed on intraoperative LU, but if postoperative laryngoscopy demonstrated normal vocal cord movement the case was assigned to the false-positive category. The same principles were equally applied for the LP method interpretation.

\section{Statistical analysis}

Statistical analysis was performed using SPSS v.23 For Windows (IBM, Armonk, NY, USA). The RLN injury incidence calculations were based on the number of nerves at risk. The McNemar test was applied to compare tests' sensitivity and specificity. The levels of significance were indicated by $p$-values. All $p$-values of less than 0.05 were considered to indicate statistical significance.

\section{Results}

\section{Clinicopathological characteristics}

One patients twelve patients were enrolled and underwent the full study protocol and in total $200 \mathrm{RLN}$ at risk were evaluated. The mean age of the patients was $56.22 \pm 13.57$ years and the majority of them were fe- male -92 (82.1\%). The indications for surgery were as follows: suspected or proven malignancy - 53 (47.3\%) cases; goiter with compressive symptoms - 39 (34.8\%) cases; and thyrotoxicosis - 20 (17.9\%) cases.

The most common operation was total thyroidectomy - 54 (48.2\%) cases (Figure 1$)$.

There were no subtotal thyroidectomies, which could underestimate the RLN palsy rate. Only 2 (1.78\%) patients underwent recurrent thyroid surgery. Histological examination showed that 72 (64.3\%) patients underwent surgery for benign pathology and 40 (35.7\%) for malignant. Papillary carcinoma accounted for the vast majority of malignant pathology cases -32 (28.6\%) (Table I).

\section{Intraoperative RLN functional status assessment}

The RLN and vagal nerve were successfully dissected and visualized in all cases. Also, in all cases, RLN and vagal nerve stimulation resulted in the same response. Visualization of at least one of the laryngeal sonographic landmarks by one of the two intraoperative LU techniques was successful in all cases.

Table I. Histological examination results

\begin{tabular}{|lc|}
\hline Result & Patients, $n$ (\%) \\
\hline Papillary thyroid carcinoma & $32(28.5)$ \\
\hline Follicular thyroid carcinoma & $4(3.5)$ \\
\hline Medullary thyroid carcinoma & $4(3.5)$ \\
\hline Nodular hyperplasia & $35(31.2)$ \\
\hline Diffuse hyperplasia & $6(5.3)$ \\
\hline Adenomatous nodules & $14(12.5)$ \\
\hline Hashimoto's thyroiditis & $1(0.9)$ \\
\hline Follicular adenoma & $16(14.6)$ \\
\hline
\end{tabular}


Table II. Laryngeal ultrasound and laryngeal palpation sensitivity, specificity, accuracy, positive and negative predictive values for intraoperative recurrent laryngeal nerve injury detection

\begin{tabular}{|lcc|}
\hline Parameter & LU (\%) & LP (\%) \\
\hline Sensitivity & 83.3 & 100 \\
\hline Specificity & 97.2 & 95.3 \\
\hline Accuracy & 96.4 & 95.5 \\
\hline Positive predictive value & 62.5 & 54.5 \\
\hline Negative predictive value & 99 & 100 \\
\hline
\end{tabular}

The RLN palsy rate based on the number of nerves at risk was 3\% and based on the number of patients was $5.4 \%$ (6 cases). VC mobility recovered in all 6 patients within 2-9 months after surgery.

Intraoperative RLN functional status assessment with LU resulted in 3 false positive cases and 1 false negative case. The LP was falsely positive in 6 cases and there were no false negative cases.

Table II shows each method's sensitivity, specificity, accuracy, positive predictive values, and negative predictive values. There was no significant difference between $L P$ and $L U$ for intraoperative RLN injury detection $(p=0.289)$.

\section{Discussion}

Our study demonstrated that LP and the new method LU are both effective for intraoperative RLN functional status evaluation. Nerve identification alone is insufficient because visual integrity does not guarantee normal RLN functional status $[7,8]$. One of the most significant attributes of neural testing is prognostication of postoperative neural function and its impact on surgical strategy in bilateral thyroid disease $[9,21,29,30]$. Accurate identification of unilateral RLN injury might allow second lobe surgery and bilateral nerve injury to be avoided. Recently, IONM has received considerable attention and has gained popularity, but controversy remains as to whether electromyographic monitoring has any clear advantage in preventing nerve injury and whether it precisely predicts VC function postoperatively [15, 31-33]. Also, it is clear that the need for specialized neural monitoring equipment increases the surgery costs and in some centers IONM is not used for this reason $[11,12,31]$. According to vari- ous authors, IONM increases the cost of surgery by $\$ 387-683[11,12]$. Also, IONM demands the precise placement of endotracheal tube electrodes over the VC, and therefore fiberoptic laryngoscopy should be used, increasing the costs even more. In contrast, LP and LU methods require the only fixed cost of a neural stimulator and an ultrasound machine.

Some studies indicate that the LP method is as reliable and sensitive as IONM in predicting RLN functional status or can be used when IONM is unavailable [19, 20, 22]. Different authors report very different rates of sensitivity and specificity of IONM for RLN injury detection. Systematic appraisal of the literature by Dralle et al. shows the sensitivity ranging from $23.5 \%$ to $100 \%$ and specificity ranging from $94 \%$ to $99.7 \%$ [34]. Two more recent studies published by Mirallie et al. [35] and Randolph et al. [36] demonstrated the low sensitivity (29\% and 33\%) and high specificity (98\% and $99.9 \%$ ) of the method. In contrast, Calo et al. reported very high values of both parameters - sensitivity $93.6 \%$ and specificity 99.4\% [37]. For comparison, the reported sensitivity of LP varies between $33.4 \%$ and $100 \%$ while specificity varies between $92.2 \%$ and $100 \%$ (Table III). Therefore, the predictive values IONM and LP can be judged as comparable.

Recent studies described a new technique, LU, to assess VC mobility and RLN functional status in preoperative and postoperative settings [23-27, 38, 39]. With regard to such advantages as simplicity, the possibility of use in combination with minimally invasive surgery and documentation of the real-time VC mobility, we considered that LU application as an intraoperative technique would be valuable to improve thyroid surgery quality. However, it was never tested in an intraoperative setting and the validity of the method was not documented before our study was conducted. The results of our study confirmed that intraoperative LU can be used for intraoperative RLN functional status assessment with a slightly higher accuracy than the technique of LP. Moreover, intraoperative LU can be a good alternative to LP in confined surgical fields, such as mini-invasive thyroidectomy or parathyroidectomy, when LP is very difficult and probably dangerous [19].

All the methods for intraoperative RLN functional status assessment require additional knowledge and skills; therefore learning curves exist. The learning curve of standardized IONM is about 50 cases $[40,41]$ and generally should be considered as long 
Table III. Reported rates of sensitivity and specificity for recurrent laryngeal nerve injury detection by intraoperative laryngeal palpation

\begin{tabular}{|c|c|c|c|c|c|c|}
\hline $\begin{array}{l}\text { Author and year } \\
\text { of study }\end{array}$ & Country & Study type & $\begin{array}{l}\text { Number } \\
\text { of patients }\end{array}$ & $\begin{array}{l}\text { Sensitivity } \\
(\%)\end{array}$ & $\begin{array}{l}\text { Specificity } \\
(\%)\end{array}$ & $\begin{array}{l}\text { Recommendations } \\
\text { for application to } \\
\text { clinical practice }\end{array}$ \\
\hline Cavicchi et al. (2018) & Italy & Retrospective cohort & 716 & 90 & 99.1 & Recommended \\
\hline Cha et al. (2018) & South Korea & Prospective cohort & 293 & 81.82 & 100 & Recommended \\
\hline Cavicchi et al. (2012) & Italy & $\begin{array}{c}\text { Prospective double } \\
\text { blind RCT }\end{array}$ & 250 & 33.4 & 97.5 & Recommended \\
\hline Cavicchi et al. (2009) & Italy & $\begin{array}{l}\text { Retrospective } \\
\text { case-control }\end{array}$ & 993 & 55.3 & 95.2 & Recommended \\
\hline Tomoda et al. (2006) & Japan & Prospective cohort & 1376 & 69.3 & 99.7 & Questionable \\
\hline Randolph et al. (2004) & USA & Prospective cohort & 449 & 100 & 100 & Recommended \\
\hline Otto et al. (2002) & USA & Retrospective cohort & 55 & 75 & 92.2 & Recommended \\
\hline
\end{tabular}

[42]. In contrast, the learning curve for transcutaneous laryngeal ultrasound is significantly shorter; surgeons could master the ultrasound after seven procedures and assess vocal cord function consistently and accurately after 40 procedures [43]. We could not assess the learning curve of intraoperative $L U$ in our study, because the procedures were performed by a single surgeon, who was already trained in perioperative LU. However, our study demonstrated that VC was visualized in all cases and the predictive values of the method were high. Therefore we assume that intraoperative LU can be handled easily without special training for surgeons already trained in perioperative LU.

The main disadvantage of LU may be a poor VC visualization rate. According to recently published studies in a perioperative setting VC cannot be visualized and assessed in approximately $20 \%$ of cases $[25,27,38]$. However, we did not experience this problem in our study, where the visualization rate was $100 \%$. To increase the VC visualization rate we used a modified LU technique - a combination of the traditional anterior approach and the novel lateral approach LU as described by Woo et al. [26]. To achieve a further increase in VC visualization rate the LU examination was defined as assessable even if 1 out of the 3 laryngeal sonographic landmarks was identified, as was suggested by Wong et al. [23]. Another potential limitation of the new intraoperative LU method is the distortion of the normal laryngeal anatomy due to the endotracheal tube position, but our study achieved a $100 \%$ visualization rate.
High specificity and a negative predictive value confirm that the observed movement on intraoperative LU after completion of one side of surgery predicts normal vocal cord function postoperatively. However, lower sensitivity and a positive predictive value make it more difficult to decide whether to continue the operation on the contralateral side when there is no visible damage to the nerve, but there is no observed movement on the intraoperative LU examination. Our experience showed that in 1 case there was no observable contraction of the cricoarytenoid muscle, yet weak movement was observed on intraoperative LU. We can speculate that remaining nerve fibers after RLN damage would produce muscular movement only on suprathreshold stimulation and too few of them were left intact to produce volitional movement on postoperative laryngeal examination. We can presume that any movement observed on intraoperative LU could be a reliable prognostic factor for temporary palsy with a reasonable prospect of recovery. All operations in our study were performed by a single surgeon, and this fact gives rise to uncertainty as to whether the same results can be reproduced in other studies.

It is difficult to draw conclusions when dealing with small numbers, and the low rates of RLN palsy may preclude the achievement of the most reliable evidence of LU sensitivity and positive predictive value.

\section{Conclusions}

Intraoperative vocal cord ultrasonography has proved feasible during thyroid surgery. It is a nonin- 
Andrius Rybakovas, Augustinas Bausys, Andrius Matulevicius, Gytis Zaldokas, Mindaugas Kvietkauskas, Gintautas Tamulevicius, Virgilijus Beisa, Kestutis Strupas

vasive tool with a very high assessability rate (100\%) when combining both anterior and lateral laryngeal ultrasonography methods and reducing the number of sonographic landmarks. LP and LU showed similar results. With regard to the slightly higher accuracy and potential application in minimally invasive surgery, LU could be recommended as the preferred technique.

\section{Conflict of interest}

\section{The authors declare no conflict of interest.}

\section{References}

1. Loyo M, Tufano RP, Gourin CG. National trends in thyroid surgery and the effect of volume on short-term outcomes. Laryngoscope 2013; 123: 2056-63.

2. Jeannon JP, Orabi AA, Bruch GA, et al. Diagnosis of recurrent laryngeal nerve palsy after thyroidectomy: a systematic review. Int J Clin Pract 2009; 63: 624-9.

3. Lifante JC, Payet C, Menegaux F, et al. Can we consider immediate complications after thyroidectomy as a quality metric of operation? Surgery 2017; 161: 156-65.

4. Hermann M, Alk G, Roka R, et al. Laryngeal recurrent nerve in jury in surgery for benign thyroid diseases: effect of nerve dissection and impact of individual surgeon in more than 27,000 nerves at risk. Ann Surg 2002; 235: 261-8.

5. Steurer M, Passler C, Denk DM, et al. Advantages of recurrent laryngeal nerve identification in thyroidectomy and parathyroidectomy and the importance of preoperative and postoperative laryngoscopic examination in more than 1000 nerves at risk. Laryngoscope 2002; 112: 124-33.

6. Dralle H, Sekulla C, Haerting J, et al. Risk factors of paralysis and functional outcome after recurrent laryngeal nerve monitoring in thyroid surgery. Surgery 2004; 136: 1310-22.

7. Bergenfelz A, Jansson S, Kristoffersson A, et al. Complications to thyroid surgery: results as reported in a database from a multicenter audit comprising 3,660 patients. Langenbecks Arch Surg 2008; 393: 667-73.

8. Snyder SK, Lairmore TC, Hendricks JC, Roberts JW. Elucidating mechanisms of recurrent laryngeal nerve injury during thyroidectomy and parathyroidectomy. J Am Coll Surg 2008; 206 123-30.

9. Goretzki PE, Schwarz K, Brinkmann J, et al. The impact of intraoperative neuromonitoring (IONM) on surgical strategy in bilateral thyroid diseases: is it worth the effort? World J Surg 2010; 34: 1274-84.

10. Malik R, Linos D. Intraoperative neuromonitoring in thyroid surgery: a systematic review. World J Surg 2016; 40: 2051-8.

11. Gremillion G, Fatakia A, Dornelles A, Amedee RG. Intraoperative recurrent laryngeal nerve monitoring in thyroid surgery: is it worth the cost? Ochsner J 2012; 12: 363-6.

12. Rocke DJ, Goldstein DP, de Almeida JR. A cost-utility analysis of recurrent laryngeal nerve monitoring in the setting of tota thyroidectomy. JAMA Otolaryngol Head Neck Surg 2016; 142: 1199-205.
13. Wojtczak B, Kaliszewski K, Sutkowski K, et al. The learning curve for intraoperative neuromonitoring of the recurrent laryngeal nerve in thyroid surgery. Langenbeck's Arch Surg 2017; 402: 701-8.

14. Hermann M, Hellebart C, Freissmuth M. Neuromonitoring in thyroid surgery: prospective evaluation of intraoperative electrophysiological responses for the prediction of recurrent laryngeal nerve injury. Ann Surg 2004; 240: 9-17.

15. Smith J, Douglas J, Smith B, et al. Assessment of recurrent laryngeal nerve function during thyroid surgery. Ann R Coll Surg Engl 2014; 96: 130-5.

16. Randolph GW, Kobler JB, Wilkins J. Recurrent laryngeal nerve identification and assessment during thyroid surgery: laryngeal palpation. World J Surg 2004; 28: 755-60.

17. Tomoda C, Hirokawa Y, Uruno T, et al. Sensitivity and specificity of intraoperative recurrent laryngeal nerve stimulation test for predicting vocal cord palsy after thyroid surgery. World J Surg 2006; 30: 1230-3.

18. Otto RA, Cochran CS. Sensitivity and specificity of intraoperative recurrent laryngeal nerve stimulation in predicting postoperative nerve paralysis. Ann Otol Rhinol Laryngol 2002; 111: 1005-7.

19. Cavicchi O, Caliceti U, Fernandez IJ, et al. The value of neurostimulation and intraoperative nerve monitoring of inferior laryngeal nerve in thyroid surgery. Otolaryngol Head Neck Surg 2009; 140: 866-70.

20. Cavicchi O, Caliceti U, Fernandez Ignacio J, et al. Laryngeal neuromonitoring and neurostimulation versus neurostimulation alone in thyroid surgery: a randomized clinical trial. Head Neck 2011; 34: 141-5.

21. Cavicchi O, Burgio L, Cioccoloni E, et al. Intraoperative intermittent neuromonitoring of inferior laryngeal nerve and staged thyroidectomy: our experience. Endocrine 2018; doi: 10.1007/ s12020-018-1739-5.

22. Cha W, Cho I, Jang JY, et al. Supramaximal neurostimulation with laryngeal palpation to predict postoperative vocal fold mobility. Laryngoscope 2016; 126: 2863-8.

23. Wong KP, Woo JW, Youn YK, et al. The importance of sonographic landmarks by transcutaneous laryngeal ultrasonography in post-thyroidectomy vocal cord assessment. Surgery 2014; 156 : 1590-6.

24. Wong KP, Lang BH, Ng SH, et al. A prospective, assessor-blind evaluation of surgeon-performed transcutaneous laryngeal ultrasonography in vocal cord examination before and after thyroidectomy. Surgery 2013; 154: 1158-64.

25. Cheng SP, Lee JJ, Liu TP, et al. Preoperative ultrasonography assessment of vocal cord movement during thyroid and parathyroid surgery. World J Surg 2012; 36: 2509-15.

26. Woo JW, Suh H, Song RY, et al. A novel lateral-approach laryngeal ultrasonography for vocal cord evaluation. Surgery 2016; 159: 52-6.

27. Carneiro-Pla D, Miller BS, Wilhelm SM, et al. Feasibility of surgeon-performed transcutaneous vocal cord ultrasonography in identifying vocal cord mobility: a multi-institutional experience. Surgery 2014; 156: 1597-602.

28. Randolph GW, Dralle H; International Intraoperative Monitoring Study G, et al. Electrophysiologic recurrent laryngeal nerve 
monitoring during thyroid and parathyroid surgery: international standards guideline statement. Laryngoscope 2011; 121 Suppl 1: S1-16.

29. Dralle H, Sekulla C, Lorenz K, et al. Loss of the nerve monitoring signal during bilateral thyroid surgery. Br J Surg 2012; 99: 1089-95.

30. Dionigi G, Frattini F. Staged thyroidectomy: time to consider intraoperative neuromonitoring as standard of care. Thyroid 2013; 23: 906-8.

31. Angelos P. Recurrent laryngeal nerve monitoring: state of the art, ethical and legal issues. Surg Clin North Am 2009; 89: 1157-69.

32. Yang S, Zhou L, Lu Z, et al. Systematic review with meta-analysis of intraoperative neuromonitoring during thyroidectomy. Int J Surg 2017; 39: 104-13.

33. Chung TK, Rosenthal EL, Porterfield JR, et al. Examining national outcomes after thyroidectomy with nerve monitoring. J Am Coll Surg 2014; 219: 765-70.

34. Dralle H, Sekulla C, Lorenz K, et al. Intraoperative monitoring of the recurrent laryngeal nerve in thyroid surgery. World I Surg 2008; 32: 1358-66.

35. Mirallié É, Caillard C, Pattou F, et al. Does intraoperative neuromonitoring of recurrent nerves have an impact on the postoperative palsy rate? Results of a prospective multicenter study. Surgery 2018; 163: 124-9.

36. Randolph GW, Kamani D. Intraoperative electrophysiologic monitoring of the recurrent laryngeal nerve during thyroid and parathyroid surgery: experience with 1,381 nerves at risk. Laryngoscope 2017; 127: 280-6.

37. Calò PG, Medas F, Conzo G, et al. Intraoperative neuromonitoring in thyroid surgery: Is the two-staged thyroidectomy justified? Int J Surg 2017; 41: S13-20.

38. Wang CP, Chen TC, Yang TL, et al. Transcutaneous ultrasound for evaluation of vocal fold movement in patients with thyroid disease. Eur J Radiol 2012; 81: e288-91.

39. Wong KP, Lang BH, Chang YK, et al. Assessing the validity of transcutaneous laryngeal ultrasonography (TLUSG) after thyroidectomy: what factors matter? Ann Surg Oncol 2015; 22: 1774-80.

40. Dionigi G, Bacuzzi A, Boni L, et al. What is the learning curve for intraoperative neuromonitoring in thyroid surgery? Int I Surg 2008; 6 suppl 1: S7-12.

41. Pragacz K, Barczynski M. Evaluation of the learning curve for intraoperative neural monitoring of the recurrent laryngeal nerves in thyroid surgery. Pol Przegl Chirur 2015; 86: 584-93.

42. Jonas J, Bähr R. Intraoperative neuromonitoring of the recurrent laryngeal nerve - results and learning curve. Zentralbl Chir 2006; 131: 443-8

43. Wong KP, Lang BH, Lam S, et al. Determining the learning curve of transcutaneous laryngeal ultrasound in vocal cord assess ment by CUSUM analysis of eight surgical residents: when to abandon laryngoscopy. World I Surg 2016; 40: 659-64.

Received: 27.10.2018, accepted: 8.11.2018 